\title{
Epidemiology, pathogens distribution, antibiotic susceptibility and the risk factors for mortality of patients with central nervous system infections: a retrospective study from 2012 to 2019 in a teaching hospital in China
}

\section{Zheng Zhang}

Shanxi Medical University

\section{Yan Song}

Second Hospital of Shanxi Medical University

Jianbang Kang

Second Hospital of Shanxi Medical University

\section{Surong Duan}

Second Hospital of Shanxi Medical University

Qi Li

Shanxi Medical University

\section{Fuqiang Feng}

Second Hospital of Shanxi Medical University Jinju Duan ( $\square$ duanjinju@163.com )

Second hospital of Shanxi Medical University

\section{Research article}

Keywords: Central nervous system infections, Pathogens distribution, Antibiotic susceptibility, Risk factors

Posted Date: March 15th, 2021

DOI: https://doi.org/10.21203/rs.3.rs-153262/v2

License: (c) (1) This work is licensed under a Creative Commons Attribution 4.0 International License. Read Full License

Version of Record: A version of this preprint was published at BMC Infectious Diseases on August 17th, 2021. See the published version at https://doi.org/10.1186/s12879-021-06561-2. 


\section{Abstract}

Background: Central nervous system (CNS) infections are relatively rare but associated with high mortality worldwide. Empirical antimicrobial therapy is crucial for the prognosis of patients with CNS infections, which should be based on the knowledge of pathogens distribution and antibiotic sensitivities. China is a vast country, and the pathogens distribution varies nationwide. The aim of this study is to investigate the features of pathogens in patients with CNS infections in north China and we tried to evaluate the risk factors for mortality.

Methods: We retrospectively analyzed the patients with positive cerebrospinal fluid (CSF) culture in a teaching hospital between January 2012 and December 2019. The following information were collected: demographic characteristics, laboratory data, causative organisms and antimicrobial susceptibility results. Univariate analysis and binary logistic regression analysis were performed to identify the risk factors for mortality.

Results: In this eight-year retrospective study, a total of 72 patients were diagnosed with CNS infections and 86 isolates were identified. Among all the microorganisms detected, Gram-positive strains consisted of $59.3 \%$, Gram-negative bacteria of $30.2 \%$ and fungi of $10.5 \%$. The predominant Gram-positive isolate was coagulase-negative staphylococci. Acinetobacter baumannii, Escherichia coli and Klebsiella were the common Gram-negative strains. Compared to 2012-2015 years, the proportion of Gram-negative bacteria increased markedly during 2016-2019 years. Vancomycin, teicoplanin and linezolid were still 100\% sensitive to Gram-positive bacteria. For the multidrug-resistant Gram-negative bacteria, only tigecycline was the $100 \%$ sensitive antibiotics. The mortality of the 72 patients was $30.6 \%$. In the multivariate analysis, age $>50$ years, combined pulmonary infection and CSF glucose $<$ normal value were associated with poor prognosis.

Conclusions: CNS infections cause high mortality worldwide. Although Gram-positive bacteria are still the primary pathogen of CNS infections, Gram-negative bacteria had increased in recent years and should be considered in the choice of empirical antibiotic treatment. Special attention should be given to older patients and those combined pulmonary infection and with low CSF glucose level.

\section{Background}

Central nervous system (CNS) infections include meningitis, encephalitis, and brain abscesses, can be complications following neurosurgical operations or occur spontaneously [1, 2]. CNS infections cause significant mortality worldwide, resulting in a poor prognosis with prolonged length of hospital stay and increasing the total cost of illness [1-5]. The pathogens causing such infections include bacteria, viruses, fungi and parasites, and it is reported that there are more than 100 pathogens that cause CNS infections [5]. Cerebrospinal fluid (CSF) specimens are commonly examined to determine the etiology of CNS infections, but the final results of the culture are often not available for $48 \mathrm{~h}$ or more $[6,7]$. Thus, empiric antimicrobial therapy is essential pending identification of the pathogen, which should be based on the 
knowledge of the prevalence of bacterial organisms and antibiotic sensitivities. However, antibiotic therapy of CNS infections has been limited due to the inefficiency of drug transport across the bloodbrain barrier (BBB) and the emergence of multidrug-resistant (MDR) strains [8-11]. In addition, the prevalence rates for pathogens causing bacterial infections vary with time, geographical distribution and underlying medical conditions [12]. China is a vast country, and the level of medical care varies nationwide, the availability of local bacterial prevalence and antibiotic sensitivities data could help to improve the antimicrobial drugs administration.

Furthermore, to explore the risk factors associated with the prognosis of patients with CNS infections would help to identify high-risk patients and provide appropriate treatment, which is of great significance for improving the patients outcomes. There were numerous studies reported the potential risk factors associated with the incidence of CNS infections in the last decades [13-15], however, data on risk factors associated with patient outcomes is limited. Herein, we present a epidemiology research of CNS infections at a large teaching hospital in north China and attempt to evaluate the crucial risk factors affecting patients outcomes following such challenging infection.

\section{Methods}

\section{Data collection}

This retrospective study was carried out at The Second Hospital of Shanxi Medical University, a 2700-bed tertiary teaching hospital in Shanxi, China. From January 2012 to December 2019, all patients with positive CSF cultures reported by the microbiological laboratory were retrospectively analyzed. Data were abstracted from the electronic medical records. For each patient, three main sets of records were collected. The first set of records related to the general demographic characteristics, including age, sex, department, comorbidity, hospital length of stay, surgery and invasive device. The second set of records concerned the pathogens isolates and their antimicrobial susceptibility results. The same strains isolated from CSF of a patient within a week were considered to be the same strains. The third set of records consisted of data related to the laboratory, as follows: glucose, protein, chlorine, adenosine deaminase (ADA), leukocyte count and erythrocyte count in CSF; white blood cell, neutrophils and percentage of neutrophils in blood; liver function indicators include alanine aminotransferase (ALT), aspartate aminotransferase (AST), total bilirubin (TBIL), alkaline phosphatase (ALP), total protein and albumin levels; renal function indicators as creatinine and urea nitrogen levels. Laboratory data was recorded as the recent data from the collection time of CSF samples.

\section{Microbiology and antimicrobial susceptibility testing}

After the CSF samples were received, centrifugation and gram stain were performed to suggest the bacterial species initially. Blood agar plates, chocolate agar plates and MacConkey agar plates were used for bacterial culture, and plates are incubated in carbon dioxide at $35^{\circ} \mathrm{C}$. Isolates were identified by the Vitek 2 automated system (Biomerieux, France). Antimicrobial susceptibility of bacteria was tested by 
Kirby-Bauer disk diffusion method and interpreted according to the latest standards of Clinical and Laboratory Standards Institute (CLSI) Guidelines.

\section{Definitions}

CNS infections was diagnosed according to the definitions of Centers for Disease Control and Prevention (CDC) as follows [16]: (1) isolation of pathogens from CSF; (2) patient was considered at least one of the following signs with no other recognized cause: fever $\left(\llbracket 38^{\circ} \mathrm{C}\right)$, headache, stiff neck, meningeal signs, cranial nerve signs, changing level of consciousness, or confusion; (3) increased white cells, elevated protein, and/or decreased glucose in CSF. MDR is defined as non-susceptibility to three or more classes of antibiotics, and extensively drug-resistant (XDR) is defined as non-susceptibility to at least one agent in all but two or fewer antimicrobial categories $[17,18]$. The study population was divided into two groups according to the final outcome of whether the patient survived.

\section{Statistical Analysis}

Data were analyzed with SPSS software version 16.0. The statistical results for continuous data were recorded as mean \pm SD or median (IQR) values according to the statistical distribution, and categorical parameters as number (\%). To compare the difference between two groups of patients (survivors and non-survivors), categorical variables were analyzed by a Chi-square test or Fisher's extract test, as required. Independent samples t-test was used for continuous variables with normal distribution, and continuous variables with non-normal distribution were compared by using the independent samples Mann-Whitney $U$ test. Two-tailed tests were used to determine statistical significance and a $p$ value $<0.05$ was considered statistically significant. Factors with a $p$ value $<0.1$ in univariate Chi-square test or Fisher's extract test were included in a binary logistic regression model to identify the independent risk factors. Laboratory data were converted to dichotomous variables by selecting a cut-off point based on the maximum or minimum referenced values.

\section{Results}

\section{Study population}

During January 2012 to December 2019, we obtained 111 positive CSF culture samples from 98 patients. Of these, 22 patients were excluded due to they did not meet the diagnostic criteria of CNS infections. In addition, 4 patients were removed because they were diagnosed with viral meningitis by the attending clinicians. Finally, 72 patients were considered with CNS infections caused by bacteria or fungi, and 86 isolates were identified. Table 1 shows the characteristics of the study population.

\section{Microbiology}

The distribution of 86 isolates is shown in table 2. Among all the microorganisms detected, Gram-positive strains consisted of $59.3 \%$, Gram-negative bacteria of $30.2 \%$ and fungi of $10.5 \%$. The predominant Grampositive isolate was Coagulase-negative staphylococci (CoNS, 43.0\%), followed by Enterococcus faecium 
(5.8\%) and Streptococcus pneumoniae (5.8\%). Acinetobacter baumannii (7.0\%), Escherichia coli (7.0\%) and Klebsiella (5.8\%) were the common Gram-negative strains. In addition, 9 fungal isolates were identified and 6 of them were Cryptococcus neoformans.

Meanwhile, the change of pathogens distribution in different periods was revealed in Fig. 1 and table 2. We can observe that the Gram-positive bacteria were still the dominant pathogens, however, the proportion of Gram-negative bacteria increased markedly from $15.4 \%$ in $2012-2015$ years to $42.6 \%$ in 2016-2019 years. Moreover, fungi were also found more frequently in the later period. Particularly, Streptococcus pneumoniae and Klebsiella were identified merely in the 2016-2019 years.

\section{Antimicrobial susceptibility testing}

Of the 86 strains, in vitro antibiotic sensitivities of Gram-positive isolates are shown in table 3. CoNS were $80 \%$ sensitive to rifampicin and $100 \%$ sensitive to vancomycin, teicoplanin and linezolid. Among the Streptococcus pneumoniae, they were all sensitive to levofloxacin, moxifloxacin vancomycin and teicoplanin. For the Enterococcus faecium, vancomycin, teicoplanin and linezolid were $100 \%$ sensitive antibiotics. Of the Gram-negative isolates, Acinetobacter baumannii were $83.3 \%$ sensitive to minocycline and no species were resistant to tigecycline. Escherichia coli were $100 \%$ sensitive to amikacin, meropenem and imipenem. For the Klebsiella pneumoniae, only tigecycline was the $100 \%$ sensitive antibiotics. The antibiotic sensitivities details of Gram-negative isolates are shown in table 4. For fungal isolates, Cryptococcus neoformans were $100 \%$ sensitive to 5 -fluorouracil, amphotericin B, fluconazole and voriconazole.

\section{Risk factors}

The demographic and clinical characteristics of both survivors and non-survivors with CNS infections are shown in Table 5. Of the study population, 50 patients survived and 22 patients died with a mortality of $30.6 \%$. In univariate analysis, we found significant differences of two groups in the following aspects: age, pulmonary infection, hypertension, CSF glucose level, CSF protein level, CSF leukocyte count, blood percentage of neutrophils, ALT, AST, serum albumin, and urea nitrogen levels. In binary logistic regression analysis, we found age $>50$ years, pulmonary infection, and CSF glucose $<$ normal value were independent risk factors for mortality. Table 6 shows the final logistic regression model.

\section{Discussion}

Microbiological examination of the CSF specimen is an important basis for the diagnosis and treatment of CNS infections. We retrospectively analyzed the patients with positive CSF culture and aimed to explore the etiology, antibiotic sensitivity and risk factors affecting the clinical outcomes of patients with CNS infections. Due to a lack of standardization of the available assays and uncertainty about sample collection and processing. False positive CSF culture can be discovered in some studies $[11,19]$. In our study, 111 positive CSF culture specimens were identified. 
According to the results of retrospective analysis, 26 samples from 26 patients were considered to be false positive in our 8-year study.

Of the 86 isolates detected from patients confirmed with CNS infections, the general pathogens distribution of Gram-positive, Gram-negative and Eumycetes in the paper were 59.3, 30.2 and 10.5\%, respectively. Which was approximate to the results of the previous studies $[11,20]$. In our study, the top seven pathogens were CoNS (43.0\%), Acinetobacter baumannii (7.0\%), Escherichia coli (7.0\%), Cryptococcus neoformans (7.0\%), Enterococcus faecium (5.8\%), Streptococcus pneumoniae (5.8\%) and Klebsiella (5.8\%). Which was not differ significantly from the multicenter study of positive CSF isolates from China Antimicrobial Surveillance Network (CHINET, www.chinets.com), as they showed that CoNS was the most frequent isolates (>44.97\%), followed by Acinetobacter baumannii (12.43\%), Klebsiella pneumoniae (8.23\%), Enterococcus faecium (3.95\%), Escherichia coli (3.92\%), Staphylococcus aureus (2.61\%) and Enterococcus faecalis (2.42\%) in 2019. Whereas, Cryptococcus neoformans accounted for a higher percentage in our study, thus fungal CNS infections should be considered when conventional empirical treatment is ineffective.

With respect to the pathogens distribution in different periods, we found that the proportion of Gramnegative bacteria increased markedly from $15.4 \%$ in $2012-2015$ years to $43.5 \%$ in $2016-2019$ years. In the last decades, Gram-negative bacteria had attracted more attention from clinicians, especially MDR/XDR Acinetobacter baumannii and Enterobacteriaceae [21-25]. In our study, Acinetobacter baumannii and Klebsiella were the significantly increased strains and the most frequent causative Gramnegative strains in the later period, accounting for $21.2 \%$ of the total isolates. Of the Gram-positive bacteria, CoNS were still the predominant organism although the percentage of it reduced dramatically from $61.5-27.7 \%$. In addition, compare to $2012-2015$ years, the species and quantities of isolated strains increased during 2016-2019 years, the Streptococcus pneumoniae and Klebsiella were identified merely in the later period. Cultivation of Streptococcus pneumoniae has been challenging because autolysis resulted in decreased viability [25], the discovery of it could be associated with the development of the laboratory and microbiological inspection techniques. The elevated microbial species in the later period might also demonstrate this opinion.

As for the results of antibiotic sensitivity in this study, Gram-positive bacteria was still $100 \%$ sensitive to vancomycin, teicoplanin and linezolid. Of them, 81.1\% (30/37) of the CoNS were methicillin-resistant strains. This finding was higher than the other studies, which were reported $75 \%$ and approximately $55 \%-75 \%$ respectively $[11,26]$. Additionally, CoNS was $80 \%$ sensitive to rifampicin. However, rifampicin is usually used to destroy the tuberculosis bacilli, monotherapy might develop drug resistance easily. The use of rifampicin as part of a combination therapy for CoNS infections of the CNS might be worth considering. Of the Streptococcus pneumoniae, 60\% (3/5) strains were resistance to penicillin in our study, the result was lower than an Ethiopian study [27], which showed that $100 \%$ resistance to penicillin. Moreover, there were several studies reported about vancomycin-resistant Enterococcus faecium [28-30], yet, no vancomycin-resistant strains were identified at the present study. 
For the Gram-negative isolates, XDR strains accounted for $20.8 \%$ of the Acinetobacter in an Indian study [31], yet, 83.3\% (5/6) of the Acinetobacter baumannii strains were XDR bacteria as we showed. An obvious distinction was the sensibility to cefoperazone-sulbactum, approximately $74 \%$ of the Acinetobacter isolates were found to be sensitive to it in Indian [31]. However, Acinetobacter baumannii strains were all resistant to it in our study. One possible cause may be the different region and medical conditions. The data presented by two Chinese hospitals about Acinetobacter baumannii indicated low sensitivity to cefoperazone-sulbactum, which were $5.3 \%, 16.3 \%$ respectively $[20,23]$. It is possibly indicated that the antibiotic resistance of Acinetobacter baumannii is relatively severe among Acinetobacter. In the meantime, meningitis caused by Enterobacteriaceae, particularly carbapenemresistant Enterobacteriaceae (CRE) remains a therapeutic challenge worldwide [24]. Among the Enterobacteriaceae strains in our study, carbapenem-resistant species appeared only in the Klebsiella. Four isolates of five Klebsiella showed resistance to carbapenems antibiotics, in which three Klebsiella pneumoniae were all carbapenem-resistant strains. With regards to the Escherichia coli, our findings presented low sensibility to cephalosporins, but they were $100 \%$ sensitive to carbapenems antibiotics. This phenomenon might demonstrated that the major resistance mechanism of the Escherichia coli in our hospital is the production of beta- lactamase enzymes rather than carbapenemases.

Antimicrobial agents have been used successfully to treat infectious diseases for a long time. Unfortunately, the misuse and overuse of antibiotics has led to increased antibiotic resistance [32]. Antimicrobial therapy for CNS infections is complicated due to the emergence of MDR strains. Particularly, for Gram-negative bacteria, tigecycline, polymyxin/colistin are usually used for the treatment of MDR/XDR Acinetobacter baumannii and Klebsiella pneumoniae infections [24, 33]. Nevertheless, due to the existence of the BBB, CNS infections does not show any improvement when treated with them by intravenous administration. Hence, the intrathecal (ITH) or intraventricular (IVT) administration of antibiotics had been performed in recent years [34-37]. Whereas, ITH and IVT antibiotic therapy has not been standardized [2]. Further work is needed to explore the antimicrobial therapy through ITH/IVT approach, this might significantly improve the outcomes of patients with CNS infections.

A previous study showed that mortality due to bacterial meningitis ranges from 10 to $20 \%$ in high resource settings and as high as $50 \%$ in lower resource settings [6]. Our study showed the mortality rate of CNS infections was $30.6 \%$ in our hospital. Therefore, it is important to determine the risk factors affecting the outcomes of patients with bacterial CNS infections, particularly in developing countries. Previous studies predicted survival following CNS infections and suggested that age $>40$ years, presence of external ventricular drainage, low CSF glucose levels, high CSF protein levels, CSF leukocyte count > $200 \mathrm{cells} / \mathrm{mm}^{3}, \mathrm{ICU}$ admission, and the presence of comorbidities were risk factors for mortality [31, 38, 39]. In our analysis, age $>50$, comorbidity (pulmonary infection) and CSF glucose $<$ normal value were independent risk factors for mortality. Older patients are usually characterised by marked altered organ and physiological functions that often requires distinct treatment, such as individual drug administration and extracorporeal therapies. Thus, comprehensive assessment of the patients status is necessary before the treatment, especially for older patients. Concurrent pulmonary infections might complicate the 
antibiotic therapy and lead to a poor prognosis at last. Low glucose level in CSF is a crucial diagnostic criterion of CNS infections. Our findings showed that this indicator is not only important indicator of diagnosis, but also crucial indicator to judge the prognosis of patients. The decreased level of CSF glucose might related to the severity of CNS infections. We believe that exploring the risk factors for mortality could help clinicians to pay particular attention to this clinical condition and improve the patients outcomes.

The present study has some limitations. First, this was a single-center study with a small sample size, characteristics that may restrict the applicability of its findings to all patients with CNS infections. Second, due to the limitation of retrospective study, drug sensitivity results were not acquired in some antibiotics. Finally, we did not analyze the cause of CNS infections, whether it was related to surgery.

\section{Conclusion}

CNS infections are serious diseases and could lead to a higher mortality rate. Although Gram-positive bacteria are still the primary pathogen of CNS infections, Gram-negative organisms had increased dramatically in recent years. For Gram-positive bacteria, vancomycin was still the $100 \%$ sensitive antibiotics and the last resort of the treatment. Unfortunately, there are great challenge for the treatment of MDR/XDR Gram-negative bacteria. Although tigecycline was $100 \%$ sensitive antibiotic, it is hard to penetrate through the BBB. ITH and IVT antibiotic therapy might be the effective treatment methods and more explore is needed. In addition, special attention should be given to older patients and those combined pulmonary infection and with low CSF glucose level.

\section{Abbreviations}

CNS: Central nervous system, CSF: Cerebrospinal fluid, BBB: blood-brain barrier, MDR: multidrug-resistant, XDR: extensively drug-resistant, ADA: adenosine deaminase, ALT: alanine aminotransferase, AST: aspartate aminotransferase, TBIL: total bilirubin, ALP: alkaline phosphatase, CLSI: Clinical and Laboratory Standards Institute, CDC: Centers for Disease Control and Prevention, CoNS: Coagulasenegative staphylococci, CRE: Carbapenem-resistant Enterobacteriaceae, ITH: intrathecal, IVT: intraventricular

\section{Declarations}

\section{Ethics approval and consent to participate}

This study was approved by the Ethics Committee of Second Hospital of Shanxi Medical University (Reference Number 2019YX-278). Due to the non-interventional retrospective nature of this study, the Ethics Committee approved an informed consent waiver for the retrospective medical record review.

\section{Consent for publication}


Not applicable.

\section{Availability of data and materials}

The data set supporting the conclusions in this article is available from the corresponding author on reasonable request.

\section{Competing interests}

The authors declare that they have no competing interests.

\section{Funding}

This study was supported by the Shanxi Province Natural Science Foundation (grant number 201901D111390). The funders had no role in study design, data collection and analysis, decision to publish, or preparation of the manuscript.

\section{Authors' contributions}

JD and FF designed the study. JK and SD collected the data. ZZ, YS, and QL analyzed and interpreted the data. ZZ and YS wrote the manuscript. All authors read and approved the final manuscript.

\section{Acknowledgements}

We thank Second Hospital of Shanxi Medical University, for supporting this research.

\section{References}

1. Giovane R, Lavender P. Central Nervous System Infections. Primary care. 2018;45(3):505-18.

2. Mrowczynski OD, Langan ST, Rizk EB. Intra-cerebrospinal fluid antibiotics to treat central nervous system infections: A review and update. Clin Neurol Neurosurg. 2018;170:140-58.

3. Yang W, Wu X, Li Z, Yuan Q, Wu G, Yu J, et al. Trends of Intra-Cranial Bacterial Infection in Patients Requiring Emergency Neurosurgery. Surg Infect (Larchmt). 2020;21(8):677-83.

4. Singhi S, Angurana SK. Principles of Management of Central Nervous System Infections. Indian J Pediatr. 2019;86(1):52-9.

5. Thanh TT, Casals-Pascual C, Ny NTH, Ngoc NM, Geskus R, Nhu LNT, et al. Value of lipocalin 2 as a potential biomarker for bacterial meningitis. Clin Microbiol Infect. 2020.

6. Poplin V, Boulware D, Bahr N. Methods for rapid diagnosis of meningitis etiology in adults. Biomark Med. 2020;14(6):459-79.

7. Hasbun R, Rosenthal N, Balada-Llasat JM, Chung J, Duff S, Bozzette S, et al. Epidemiology of Meningitis and Encephalitis in the United States, 2011-2014. Clin Infect Dis. 2017;65(3):359-63. 
8. Lewin JJ 3rd, Cook AM, Gonzales C, Merola D, Neyens R, Peppard WJ, et al. Current Practices of Intraventricular Antibiotic Therapy in the Treatment of Meningitis and Ventriculitis: Results from a Multicenter Retrospective Cohort Study. Neurocrit Care. 2019;30(3):609-16.

9. Nau R, Blei C, Eiffert H. Intrathecal Antibacterial and Antifungal Therapies. Clinical Microbiology Reviews. 2020;33(3).

10. Zimmer AJ, Burke VE, Bloch KC. Central Nervous System Infections. Microbiology Spectrum. 2016;4(3).

11. Chang JB, Wu H, Wang H, Ma BT, Wang RZ, Wei JJ. Prevalence and antibiotic resistance of bacteria isolated from the cerebrospinal fluid of neurosurgical patients at Peking Union Medical College Hospital. Antimicrob Resist Infect Control. 2018;7:41.

12. Chang C, Ye J, Yang C, Huang P, Chiang P, Lee M. Influence of third-generation cephalosporin resistance on adult in-hospital mortality from post-neurosurgical bacterial meningitis. Journal of microbiology, immunology, and infection = Wei mian yu gan ran za zhi. 2010;43(4):301-9.

13. Wang LY, Cao XH, Shi LK, Ma ZZ, Wang Y, Liu Y. Risk factors for intracranial infection after craniotomy: A case-control study. Brain Behav. 2020;10(7):e01658.

14. Zhan R, Zhu Y, Shen Y, Shen J, Tong Y, Yu H, et al. Post-operative central nervous system infections after cranial surgery in China: incidence, causative agents, and risk factors in 1,470 patients. Eur $\mathrm{J}$ Clin Microbiol Infect Dis. 2014;33(5):861-6.

15. Bodilsen J, Dalager-Pedersen M, van de Beek D, Brouwer MC, Nielsen H. Risk Factors for Brain Abscess: A Nationwide, Population-Based, Nested Case-Control Study. Clin Infect Dis. 2020;71(4):1040-6.

16. Horan TC, Andrus M, Dudeck MA. CDC/NHSN surveillance definition of health care-associated infection and criteria for specific types of infections in the acute care setting. Am J Infect Control. 2008;36(5):309-32.

17. Magiorakos AP, Srinivasan A, Carey RB, Carmeli Y, Falagas ME, Giske CG, et al. Multidrug-resistant, extensively drug-resistant and pandrug-resistant bacteria: an international expert proposal for interim standard definitions for acquired resistance. Clin Microbiol Infect. 2012;18(3):268-81.

18. Diamantino-Miranda J, Aguiar SI, Carrico JA, Melo-Cristino J, Ramirez M. Clonal and serotype dynamics of serogroup 6 isolates causing invasive pneumococcal disease in Portugal: 1999-2012. PLoS One. 2017;12(2):e0170354.

19. Wong PH, Maranich AM, Muench DF. Isolation of bacterial cerebrospinal fluid culture contaminants at a major military medical center. Diagn Microbiol Infect Dis. 2013;77(4):357-61.

20. Tian L, Zhang Z, Sun ZY. Pathogen Analysis of Central Nervous System Infections in a Chinese Teaching Hospital from 2012-2018: A Laboratory-based Retrospective Study. Curr Med Sci. 2019;39(3):449-54.

21. Pandey S, Li L, Deng XY, Cui DM, Gao L. Outcome Following the Treatment of Ventriculitis Caused by Multi/Extensive Drug Resistance Gram Negative Bacilli; Acinetobacter baumannii and Klebsiella pneumonia. Front Neurol. 2018;9:1174. 
22. Rodriguez Guardado A, Blanco A, Asensi V, Perez F, Rial JC, Pintado V, et al. Multidrug-resistant Acinetobacter meningitis in neurosurgical patients with intraventricular catheters: assessment of different treatments. J Antimicrob Chemother. 2008;61(4):908-13.

23. Liang W, Yuan-Run Z, Min Y. Clinical Presentations and Outcomes of Post-Operative Central Nervous System Infection Caused by Multi-Drug-Resistant/Extensively Drug-Resistant Acinetobacter baumannii: A Retrospective Study. Surg Infect (Larchmt). 2019;20(6):460-4.

24. Guanghui Z, Jing L, Guojun Z, Hong L. Epidemiology and risk factors of neurosurgical bacterial meningitis/encephalitis induced by carbapenem resistant Enterobacteriaceae. J Infect Chemother. 2020;26(1):101-6.

25. Petti CA, Woods CW, Reller LB. Streptococcus pneumoniae antigen test using positive blood culture bottles as an alternative method to diagnose pneumococcal bacteremia. J Clin Microbiol. 2005;43(5):2510-2.

26. Piette A, Verschraegen G. Role of coagulase-negative staphylococci in human disease. Vet Microbiol. 2009;134(1-2):45-54.

27. Assegu Fenta D, Lemma K, Tadele H, Tadesse BT, Derese B. Antimicrobial sensitivity profile and bacterial isolates among suspected pyogenic meningitis patients attending at Hawassa University Hospital: Cross-sectional study. BMC Microbiol. 2020;20(1):125.

28. Le J, Bookstaver PB, Rudisill CN, Hashem MG, Iqbal R, James CL, et al. Treatment of meningitis caused by vancomycin-resistant Enterococcus faecium: high-dose and combination daptomycin therapy. Ann Pharmacother. 2010;44(12):2001-6.

29. Jaspan HB, Brothers AW, Campbell AJ, McGuire JK, Browd SR, Manley TJ, et al. Multidrug-resistant Enterococcus faecium meningitis in a toddler: characterization of the organism and successful treatment with intraventricular daptomycin and intravenous tigecycline. Pediatr Infect Dis J. 2010;29(4):379-81.

30. Inan D, Gunseren F, Colak D, Saba R, Kazan S, Mamikoglu L. First confirmed case of vancomycinresistant Enterococcus faecium meningitis in Turkey: case report and literature review. $\mathrm{J}$ Chemother. 2004;16(6):608-11.

31. Sharma R, Goda R, Borkar SA, Katiyar V, Agarwal S, Kumar A, et al. Outcome following postneurosurgical Acinetobacter meningitis: an institutional experience of 72 cases. Neurosurgical Focus. 2019;47(2).

32. Li L, Zhu C, Huang H. Clinical epidemiology and outcomes of biliary tract infections caused by Klebsiella pneumoniae. Ann Transl Med. 2019;7(14):304.

33. Chang JB, Chen Y, Wang H, Ma X, Zhang X, Wu H, et al. Combined Strategy for Post-Operative Patients with Central Nervous System Infections Caused by Extensively Drug-Resistant/Pan-DrugResistant Acinetobacter baumannii: A Retrospective Study. Surg Infect (Larchmt). 2020;21(10):8538.

34. Velkov T, Dai C, Ciccotosto GD, Cappai R, Hoyer D, Li J. Polymyxins for CNS infections: Pharmacology and neurotoxicity. Pharmacol Ther. 2018;181:85-90. 
35. Michalopoulos A, Karatza D. Multidrug-resistant Gram-negative infections: the use of colistin. Expert review of anti-infective therapy. 2010;8(9):1009-17.

36. Tsuji BT, Pogue JM, Zavascki AP, Paul M, Daikos GL, Forrest A, et al. International Consensus Guidelines for the Optimal Use of the Polymyxins: Endorsed by the American College of Clinical Pharmacy (ACCP), European Society of Clinical Microbiology and Infectious Diseases (ESCMID), Infectious Diseases Society of America (IDSA), International Society for Anti-infective Pharmacology (ISAP), Society of Critical Care Medicine (SCCM), and Society of Infectious Diseases Pharmacists (SIDP). Pharmacotherapy. 2019;39(1):10-39.

37. Li LM, Zheng WJ, Shi SW. Spinal arachnoiditis followed by intrathecal tigecycline therapy for central nervous system infection by extremely drug-resistant Acinetobacter baumannii. J Int Med Res. 2020;48(7):300060520920405.

38. Mao DH, Miao JK, Zou X, Chen N, Yu LC, Lai X, et al. Risk Factors in Predicting Prognosis of Neonatal Bacterial Meningitis-A Systematic Review. Front Neurol. 2018;9:929.

39. Shi YJ, Zheng GH, Qian LY, Qsman RA, Li GG, Zhang GJ. Longitudinal Analysis of Risk Factors for Clinical Outcomes of Enterobacteriaceae Meningitis/Encephalitis in Post-Neurosurgical Patients: A Comparative Cohort Study During 2014-2019. Infect Drug Resist. 2020;13:2161-70.

\section{Tables}

Due to technical limitations, the tables are only available as a download in the supplemental files section.

\section{Figures}




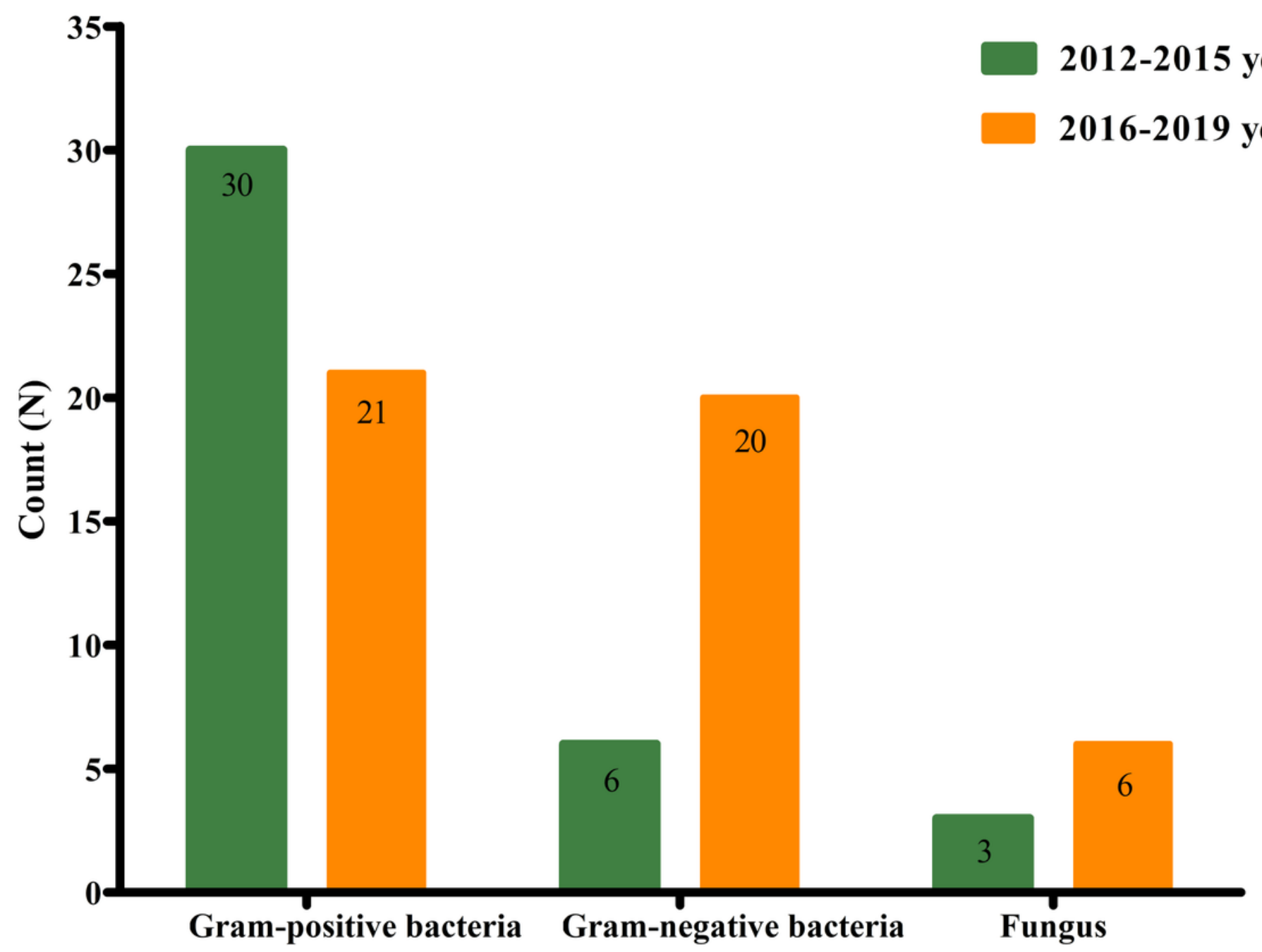

Figure 1

Change of pathogens distribution in different periods

\section{Supplementary Files}

This is a list of supplementary files associated with this preprint. Click to download.

- Table1.xlsx

- Table2.xlsx

- Table3.xlsx

- Table4.xlsx

- Table5.xlsx

- Table6.xlsx 